\title{
Age-related declines in general cognitive abilities of Balb/C mice are associated with disparities in working memory, body weight, and general activity
}

\author{
Louis D. Matzel, ${ }^{1}$ Henya Grossman, Kenneth Light, David Townsend, \\ and Stefan Kolata \\ Department of Psychology, Program in Behavioral Neuroscience, Rutgers University, Piscataway, New Jersey 08854, USA
}

\begin{abstract}
A defining characteristic of age-related cognitive decline is a deficit in general cognitive performance. Here we use a testing and analysis regimen that allows us to characterize the general learning abilities of young (3-5 mo old) and aged (19-21 mo old) male and female Balb/C mice. Animals' performance was assessed on a battery of seven diverse learning tasks. Aged animals exhibited deficits in five of the seven tasks and ranked significantly lower than their young counterparts in general learning abilities (aggregate performance across the battery of tasks). Aging added variability to common core performance (i.e., general learning ability), which translated into increased variability on the individual cognitive tasks. Relatedly, general learning abilities did not differ between the two ages among the best quartile of learners (i.e., cognitive abilities were spared in a subsample of the aged animals). Additionally, working memory capacity (resistance to interference) and duration (resistance to decay) accounted for significantly more of the variability in general learning abilities in aged relative to young animals. Tests of 15 noncognitive performance variables indicated that an increase in body weight (and an associtaed decrease in general activity) was characteristic of those aged animals which exhibited deficient general learning abilities. These results suggest the possibility that general cognitive deficits in aged animals reflect a failure of specific components of the working memory system, and may be related to variations in body weight and an associated decrease in activity.
\end{abstract}

Broad and general learning deficits are a defining feature of the cognitive phenotype of elderly humans. However, investigations of the neurological basis of age-related cognitive decline (particularly in nonhuman animals) have typically focused on specific types or classes of cognitive abilities. Although the effects of aging on hippocampus-dependent cognitive processes have been well documented (for reviews, see Gallagher and Rapp 1997; Rosenzweig and Barnes 2003), and multivariate approaches to the assessment of the impact of aging on noncognitive classes of behavior have been reported (e.g., Markowska and Breckler 1999), systematic efforts to describe learning impairments that transcend single domains of cognitive abilities have been rare. Notwithstanding the success of efforts to characterize the impact of aging on cognitive "domains," the lack of attention to domain-independent (i.e., "general") cognitive abilities limits the functional application of these conclusions. The absence of animal studies of age-related declines in general cognitive abilities is especially problematic in light of data obtained from human test batteries which indicate that age-related learning deficits transcend specific domains of learning and are expressed independently of the sensory, motor, motivational, or information processing demands of individual learning tasks. It has been estimated that as much as $25 \%-50 \%$ of the age-related decline in cognitive/learning test performance is attributable to a perturbation of this influence on general cognitive abilities (Sternberg 1997; Sternberg and Kaufman 1998; Plomin 1999). Furthermore, the proportion of variance between individuals that is accounted for by general abilities increases across the life span, accounting for as much as $80 \%$ of the variance between elderly individuals

\footnotetext{
1 Corresponding author.
}

E-mail matzel@rci.rutgers.edu; fax (732) 445-2263.

Article is online at http://www.learnmem.org/cgi/doi/10.1101//m.954808. and only $40 \%$ ( $\pm 10 \%$ ) among the rest of the population (Plomin and Spinath 2002). Despite the critical need to elucidate the basis for the impairment of general abilities, studies of age-related learning abilities in laboratory animals most often depend on single, limited, or idiosyncratic learning tasks (Gage et al. 1984; Barnes and McNaughton 1985; Markowska et al. 1994; Meliska et al. 1997; Nalbantoglu et al. 1997; Vogel et al. 2002). Absent a strategy that is specifically sensitive to the general cognitive abilities of aged animals, a high proportion of the cognitive deficits that accrue with aging may go unexplained.

Establishing an animal model of age-related declines in general cognitive abilities could provide a foundation from which to elucidate the underlying basis of this pervasive impairment. It has recently been proposed that a specific decline in processing capacity (i.e., working memory capacity) is responsible for much of the broad decline in cognitive function that accrues with age (Salthouse et al. 2003). This hypothesis is based on the broadly held belief that components of the working memory system are recruited to accomplish (to varying degrees) any cognitive task, and thus are a critical determinant of general (cf. "fluid") intelligence (Conway and Engle 1996). We have begun to explore this possibility in young, genetically diverse mice, and have determined that processing components of the working memory system (i.e., working memory capacity and selective attention) significantly covary with general learning abilities, while storage components of working memory (i.e., short-term memory duration and working memory span) do not (Kolata et al. 2005, 2007). We further addressed this issue here, first by comparing the general learning abilities of young (3-5 mo old) and aged (19-21 mo old) Balb/C mice, and by determining the extent to which general learning abilities covary with working memory capacity and span in each of the two age groups.

Here, we assessed general learning abilities with a battery of 
seven learning tasks (Lashley Maze, passive avoidance, fear conditioning, odor discrimination, spatial water maze, spatial radial arm maze, and reinforced alternation) that make distinct sensory, motor, and motivational demands on the animals and which depend on diverse information processing strategies and presumed neuroanatomical systems. We then assessed the efficacy of working memory capacity and span. Lastly, we administered 15 tests of basic sensory and motor acuity as well as native behavioral tendencies related to emotionality and stress responsivity. Subsequent to behavioral testing, principal component factor analyses were applied to the data, allowing us to identify factors which describe animals' performance across all learning tasks and, furthermore, to estimate the contribution of variations in components of the working memory system as well as variations in sensory/motor performance and emotionality to general learning abilities. With these testing and analysis protocols, it was possible to precisely describe the general learning abilities of individual animals and to assess differences in general learning abilities across populations, e.g., young vs. old subjects, as well as any potential contributors to these differences.

\section{Materials and Methods}

\section{Subjects}

A total of $56 \mathrm{Balb} / \mathrm{C}$ mice obtained from the NIA breeding facility were used in this experiment. The inbred Balb/C strain (as opposed to an outbred strain) was appropriate for this study as our primary intent was to examine differences between groups (i.e., ages). This more genetically homogeneous strain, while reducing variations between individuals, maximizes our sensitivity to differences between groups. All mice were obtained in a single shipment and arrived in our laboratory at $2.5 \mathrm{mo}(n=30 ; 16$ female, 14 male) or $18.5 \mathrm{mo}$ ( $n=30$; 16 female, 14 male) of age. Two of the aged male mice became ill (and subsequently died) during the course of testing (and were excluded from all analyses), resulting in a final $n$ of 28 in the aged sample. At the start of behavioral testing, young mice ranged from 17.7 to $30.8 \mathrm{~g}$, and aged mice from 25.7 to $40.3 \mathrm{~g}$. All animals were housed individually in clear shoebox cages in a temperature- and humiditycontrolled colony room and were maintained on a 12-h light/ dark cycle. In order to minimize defensive behaviors (which can impede goal-directed behaviors) that often occur in response to handling, prior to the start of the experiment all animals were handled by an experimenter (removed from their home cages and held by an experimenter) for $90 \mathrm{sec} /$ day, five days/week over an 18-d period prior to the start of behavioral testing. Behavioral testing required $\sim 10 \mathrm{wk}$ to complete, such that testing of young and aged mice was concluded at $\sim 5$ and 21 mo of age (respectively).

\section{Food and water deprivation}

For the cognitive tasks that required food deprivation, ad lib food was removed from the animals' home cages at the end of the light cycle $\sim 40 \mathrm{~h}$ prior to the start of training (and thus encompassing the "rest" day between successive tasks). During the deprivation period, animals were provided food in their home cages for $90 \mathrm{~min} /$ day during the last $2 \mathrm{~h}$ of the light cycle, and thus were $\sim 16 \mathrm{~h}$ food-deprived at the time of training or testing. This deprivation schedule was deemed "mild" (animals typically lost $<5 \%$ of their free-feeding body weight during this period) but was sufficient to maintain stable performance on these tasks. In the one task that required water deprivation, the same schedule was followed except that free access to water was limited to $60 \mathrm{~min}$ per day.

\section{Behavioral training and testing}

To quantify individual differences in learning among mice, a variant of the procedures previously reported was used (Matzel et al. 2003, 2006; Kolata et al. 2005, 2007, 2008). All animals were tested in a series of six independent learning tasks (Lashley III Maze, passive avoidance, spatial water maze, odor discrimination, and fear conditioning) that place unique sensory, motor, motivational, and information processing demands on the animals. Briefly, passive avoidance is an operant conditioning paradigm in which the animals must learn to be passive in order to avoid aversive light and noise stimulation. The spatial water maze encourages the animals to integrate spatial information to efficiently escape from a pool of water. Odor discrimination is a task in which animals must discriminate and use a target odor to guide their search for food. In the reinforced alternation task, animals must alternate between left and right arms in a T-maze to obtain food. In the radial arm maze, animals rely on spatial cues to efficiently choose between eight arms in which a food reward is located at the arms end. And fear conditioning (assessed by suppression of an ongoing behavior) is a conditioning test in which the animals learn to associate a tone with a shock. In addition to tests of learning acquisition, all animals were tested in variants of the radial arm maze that allowed us to isolate the efficacy of the animals' working memory span (rate of decay) and working memory capacity (resistance to interference). Testing order was designed so that tasks that impinge on similar information processing demands or motor requirements were separated (in the testing order) to the greatest possible extent.

\section{Tests of learning and working memory}

\section{Lashley III Maze (LM)}

This maze consists of a start box, three interconnected alleys, and a goal box. Previous studies have shown that, over successive trials, the latency and number of errors to find the goal decrease. When extra-maze cues are minimized, the animals tend to use egocentric methods (e.g., fixed motor patterns) to locate the goal box.

A LM scaled for use with mice (see Matzel et al. 2003) was constructed from black Plexiglas and located in a dimly lit room (10 Lux at the floor of the maze). A 3-cm-diameter white circle was located in the center of the goal box, and 45-mg Bio-Serv food pellets (dustless rodent grain) were placed in the cup to motivate the animal's behavior.

On two successive days, food-deprived animals received a day of acclimation to the maze, followed by a single training day. Prior to the day of acclimation, all animals received three pellets of the reinforcer in their home cage. On the acclimation day, each mouse was confined in each of the first two alleys of the maze for 4 $\mathrm{min}$, and in the final alley (containing the goal box) for $6 \mathrm{~min}$. On this acclimation day, three pellets were placed in the goal box. At the end of each period, the animal was physically moved to the next alley. This acclimation exposure was intended to adapt the animals to the apparatus prior to actual training. On the subsequent training day, each animal was placed in the start box and allowed to freely navigate the maze, during which time their latency to locate their food and the number of errors were recorded. (An error was constituted by either a wrong turn or a retracing of the animal's path.) Upon locating and consuming the food pellet, the animal was returned to its home cage for a 25-min intertrial interval (ITI), during which time the maze was cleaned. (In this and other multi-trial tasks, long intertrial intervals were used, as our previous work has determined that the longer ITI supports more stable acquisition of the learned response.) The animals completed five trials during the single training. For the purpose of characterizing individual mice for 
the principal component analysis, the number of errors committed by each animal on Trials 3 and 4 was averaged and served as each animal's index of performance.

\section{Passive avoidance (PA)}

In this assay, animals learn to suppress their exploratory tendency in order to avoid aversive stimuli. The animals are placed on a platform and, when they step down, are exposed to an aversive stimulus compound comprised of a bright light and loud oscillating tone (i.e., "siren").

A chamber with a white grid floor $16 \times 12 \mathrm{~cm}$ (length $\times$ width) that is illuminated by a dim red light was used for both acclimation and testing. An enclosed platform $(70 \times 45 \times 45 \mathrm{~cm}$, length $\times$ width $\times$ height) constructed of black Plexiglas and elevated $5 \mathrm{~cm}$ above the grid floor was located at the back of the chamber. It has only one opening facing the grid floor, which allows the animal to step down onto the floor. The exit from the platform can be blocked remotely by a clear Plexiglas guillotinestyle door. When an animal leaves the platform and makes contact with the grid floor, it initiates the aversive stimuli.

Animals are placed on the platform with the door closed, confining them in the enclosure. After $5 \mathrm{~min}$, the door is opened and the latency of the animal to leave the platform and make contact with the floor was recorded. After they make contact, the aversive stimuli are initiated and the platform is lowered, exposing them to the stimuli for $4 \mathrm{sec}$, after which they are allowed access to the enclosure again. They were then again confined on the platform for $5 \mathrm{~min}$, after which the door opened and their latency to walk onto the grid floor was recorded for a second time. For the purpose of principal component analysis, the ratio of the post-training step latency to pretraining step latency served as the index of performance.

\section{Water maze (WM)}

This task requires the animals to locate a submerged platform in a pool of opaque water (from which they are motivated to escape). Absent distinct inter-maze cues, animals' performance in this maze is highly dependent on their integration of spatial cues (Morris 1981). The latency and the path length to locate the platform decrease over successive trials, despite entering the pool from different locations on each trial.

A round pool (140 cm diameter, $56 \mathrm{~cm}$ deep) was filled to within $20 \mathrm{~cm}$ of the top with water that is clouded with a nontoxic, water soluble black paint. A hidden 14-cm-diameter black platform was located in a fixed position $1 \mathrm{~cm}$ below the surface of the water. The pool was enclosed by a ceiling-high black curtain on which five different light patterns (which served as spatial cues) were fixed at various positions. These light cues provided the only illumination of the maze, which was $16 \mathrm{fc}$ at the water's surface.

On the day prior to training, each animal was confined to the platform by a clear Plexiglas cylinder that fits around the platform for $360 \mathrm{sec}$. On the next two training days, the animals were started from one of three positions for each trial such that no two subsequent trials start from the same position. The animal is said to have successfully located the platform when it remains on the platform for $10 \mathrm{sec}$. After locating the platform or swimming for $90 \mathrm{sec}$, the animals were left or placed on the platform for $10 \mathrm{sec}$, after which they were removed for $10 \mathrm{~min}$ and placed in a holding box before the start of the next trial. Each animal completed 11 total trials (six on the first training day, five on the second). The latency and path (obtained with a videotracking device) to find the platform were recorded for each trial. For measurements of path length, a ratio of the optimal path length (the length of a straight line between the start location and the escape platform) relative to the actual path taken by each animal length was computed. (Since starting locations [and thus the actual length of the optimal path] differ on each trial, this measure provides a slightly more accurate index of the animals' performance than would absolute path lengths.) Each animal's performance on Trials 5 and 6 was averaged and served as that animal's index of learning for purposes of principal component analysis. After the 11th training trial, animals were returned to their home cages for $3 \mathrm{~h}$ and were then administered a 30 -sec "probe" trial in which the escape platform was removed from the maze and the percent of time spent searching in the target quadrant was recorded.

\section{Associative fear conditioning (FC)}

Animals received a tone (CS) paired with a mild foot shock (US). To measure the conditioned fear responses, the lick rates (of water-deprived animals) were assessed prior to and during the presentation of the tone CS.

Two distinct experimental chambers were used (a training context and a testing context) to avoid interactions between the training context and the CS at the time of testing. Each $32 \times 32 \times 28$-cm box (lenght $\times$ width $\times$ height) was contained within a sound- and light-attenuating chamber. The training box was brightly lit with clear Plexiglas walls, no lick tube, and had a stainless-steel grid floor ( $5 \mathrm{~mm}$ spacing). The test chamber was dimly lit and the walls were covered with an opaque pattern of alternating black and white vertical stripes and the floor was formed in a grid of stainless $1.5 \mathrm{~m}$ rods arranged in $8 \mathrm{~mm}$ squares. A water lick tube protruded through a small hole in one wall of the box. In the training chamber a 0.6-mA constant current foot shock could be delivered through the floor and a $40-\mathrm{dB}_{\mathrm{c}}$ tone (Sonalert) could be delivered through a speaker mounted at the center of the chamber's ceiling.

Water-deprived animals were acclimated to the training and testing contexts by placing each animal in both boxes for 30 min on the day before training. Training on the subsequent day occurred in a single 40-min session during which the animals received two tone-shock pairings after $15 \mathrm{~min}$ and after $30 \mathrm{~min}$. They were then returned to their home cages for $60 \mathrm{~min}$ after which they were reacclimated to the test chamber for $20 \mathrm{~min}$. The following day (test day) the animals were placed in the testing chamber and, after completion of 25 licks, the CS was presented and stayed on until the animal completed an additional 25 licks or times out at $600 \mathrm{sec}$. The ratio of the time to complete 25 licks prior to the CS presentation to the time to complete 25 licks during the CS presentation served as an index of learning.

\section{Odor discrimination (OD)}

Rodents are adept at using odor to guide their reinforced behavior. This task is modified from one developed by Sara et al. (1999) but scaled for use with mice. In this task, mice navigate through a field using unique odors to guide them. The animals learn to choose the food cup that contains the target smell when given three choices. The food cup locations are rearranged on each trial but the accessible food is always marked by the same target odor (in this case, mint).

The odor discrimination chamber consisted of a black Plexiglas $60-\mathrm{cm}$ square field with $30-\mathrm{cm}$ high walls, which was located in a dimly lit room with good ventilation. Three aluminum food cups were placed in three corners. Only the target cup had the food accessible. The other two cups had food located in a covered hole drilled into the side with a ventilation hole allowing the mice to smell the food but not access it. One 30-mg portion of chocolate-flavored puffed rice acted as a reinforcer and was placed in a depression on top of the target cup. A cotton-tipped laboratory swab ( $2 \mathrm{~cm}$ long) was loaded before each trial with 25 il of lemon-, mint-, or almond-flavored extract and extended 
vertically from the back corner of each cup. Mint was always the target odor. In some replications, in lieu of aluminum food cups, movable $30 \times 15$-cm walls (height $\times$ length) with attached food cups and slots for the cotton swaps were used. The animals had to go behind the movable walls in order to reach the food cups and hence find the rewards.

Each animal had one day of acclimation and one day of testing. The night prior to the acclimation day, food was removed from each animal's home cage. The next day each mouse was placed in the box for $20 \mathrm{~min}$ without the cups. At the end of the day each animal received three pieces of the reinforcer in their home cage. On the training day each animal received four trials in which they were placed in the corner of the training chamber which did not contain a food cup. On the first trial an additional reinforcer was placed on the edge of the target cup (mint). At the end of each trial the food cups were rearranged but mint always remained as the target odor. For each trial both latency to locate the food and number of errors were recorded (where an error is making contact with or sniffing within $2 \mathrm{~cm}$ of an incorrect food cup). On replications, which used removable walls instead of aluminum food cups, an error was counted when an animal went behind the movable wall. For purpose of the principal component analysis, the average number of errors across Trials 2 and 3 served to index each animal's learning performance.

\section{Reinforced alternation (RA)}

In this task, animals learn to alternate between entering one of two arms that intersected to form the top of a "T." On each trial a food reinforcer was present at the end of one arm. The location of the reinforcer shifted to the alternate arm after each successful retrieval of food. In order to perform efficiently in such a task, the animals had to alternate choices on successive trials (win-shift) to minimize the amount of effort it required to locate the food.

The apparatus consisted of a start arm $(7.5 \mathrm{~cm}$ wide $\times 17 \mathrm{~cm}$ long) that intersected in the middle of an alley that forms the top of a " $\mathrm{T}$ " (92 cm long $\times 6 \mathrm{~cm}$ wide). The entire maze was enclosed in a 5-cm-high wall. The initial segment of the start arm was segregated by a guillotine door that is remotely operated by the experimenter. This segment of the arm constituted the start box. At the entry of each choice arm there was another experimenteroperated door. On the wall of the right arm there were vertical white strips an inch apart. On the wall of the left arm there were vertical strips. These strips will be used to help the animal discriminate between the arms.

Training was conducted over two consecutive days. On Day 1 , animals were acclimated to the maze and allowed to make four forced choices. On the first exposure, the animal was held in the start box for $30 \mathrm{sec}$, after which it was allowed to traverse the maze; the door into the left arm was locked closed, and the right door was open. A 14-mg Bio-Serv pellet (dustless rodent grain) was located in the food cup in the right arm. After consuming the food, the animal was returned to the start box for a 20-sec ITI. On the second exposure, this procedure was repeated, but the right door was locked and the left door open. After a 20-sec ITI, this sequence was repeated for two additional exposures. Through this sequence of four forced choices, the animals were acclimated to the maze.

On the subsequent day, animals were trained. On all training trials, each choice door was fully open. On Trial 1, a reinforcer was available in both food cups and the animal could make a free choice. On the second trial, reinforcement was available in the arm not entered on the first trial. If an animal chose the correct arm, the location of the reinforcer alternated on the following trial. If an incorrect choice was made, the animal was allowed to correct its mistake and locate the food in the other arm. In either case, after the reinforcer was consumed, the animal was placed back in the start box to begin a 20-sec ITI. Animals' choices were recorded on each trial for 12 trials.

\section{Assessment of working memory span and capacity}

To assess differences between animals in the efficacy of their working memory, the animals were trained to asymptotic levels of performance on two distinct eight-arm radial mazes ("primary" and "secondary"). The two mazes were located in the same testing room, and thus shared an overlapping set of extramaze visual cues. (The center points of each maze were displaced by 96 $\mathrm{cm}$, and the location of each maze remained constant across all training and testing sessions.) The primary maze was constructed of black Plexiglas and contained a closed central hub with experimenter-operated doors that could confine animals between successive arm choices. The secondary maze had no doors on the center hub and was composed of gray Plexiglas. After completion of the eight trials used to assess acquisition in the black (primary) maze, animals received four additional trials in that maze (for a total of 12 trials) and 12 trials in the gray (secondary) maze. The final four trials in the black maze were conducted after every third trial in the gray maze.

After 12 training trials, all animals were performing at a criterion indicative of asymptotic performance (an average of four or fewer errors/trial to retrieve all reinforcers over the final two training trials). As a consequence, variations in spatial learning abilities were mitigated during the subsequent tests of working memory. This was confirmed by the observation that there was no correlation between the animals' asymptotic performance on the 12th training trial and their general learning ability. Furthermore, young and old animals did not differ in their performance on the final trial in the primary maze, $t_{(56)}=1.12$, not significant (ns).

After asymptotic performance in the radial arm mazes was attained, two manipulations were introduced to assess the efficiency of animals' working memory. In the first manipulation (working memory span), mice were allowed to make four correct choices in the primary maze before being confined to the center compartment for a fixed amount of time $(60 \mathrm{sec})$, released, and then allowed to collect the final four food pellets. In the second manipulation (working memory capacity), animals were required to perform alternately (within a trial) in both the primary and secondary mazes. After three choices in the primary maze, each animal was confined to its center compartment, removed, and placed in the secondary maze. There it was allowed to make three additional choices (where in no instance did an animal commit an error). Following the three choices in the secondary maze, the animal was returned to the primary maze where it was allowed to perform until it had made three correct choices. The animal was then confined to the center compartment, removed, placed in the secondary maze, and allowed to perform until it made three correct choices. The animal was then returned to the primary maze, where it was allowed to perform until the final two reinforcers were located and consumed. On the day following such a test trial, all animals received a standard training trial in the primary maze to ensure stable performance in that maze (and to provide a behavioral baseline for comparison to performance on tests of working memory).

In both the delay (span) and the interference (capacity) manipulations, working memory was assessed by comparing the number of errors each animal made in locating the last two available rewards in the primary maze on each trial relative to the number of errors it committed to locate the last two rewards on baseline trials (defined as the average number of errors on Trial 12 of training and the two standard trials that intervened working memory tests). The majority of animals' errors were committed during these last two choices, therefore this criterion was 
chosen because it was sensitive to the greatest differences between animals.

\section{Tests of unlearned behaviors and fitness}

All animals were assessed on tests of exploration, emotionality, sensory/motor abilities, and fitness. Each of the following tests was administered with one day intervening between the completion of one test and the start of the subsequent test. Behavior in the novel open field (activity, exploration, and fecal boli; Task 1) was assessed two days prior to the start of tests of learning; with the exception of body weights and swim speeds, all other assessments (including additional tests of exploration) were made during a 16-d period beginning six days after the completion of the learning battery and tests of working memory.

In all but several instances, tests of unlearned behaviors and fitness were completed in a single day. Many tests yielded several different measures of performance such that 15 variables were assessed that were relevant to balance, strength, coordination, general activity, exploratory tendencies, fear and anxiety responses, response to novelty, and pain sensitivity. The apparatus and parameters that are described below had been chosen based on prior work (Matzel et al. 2006; Grossman et al. 2007) in which they were determined to be adequate to capture a wide range of variations in performance across individual animals.

\section{Open field exploration}

A square field $(46 \times 46 \mathrm{~cm})$ with 13 -cm-high walls was constructed of white Plexiglas and was located in a brightly lit room (400 Lux) with a background noise of $65 \mathrm{~dB}_{\mathrm{c}}$. The field was conceptually comprised of a $6 \times 6$ grid $(7.65-\mathrm{cm}$ quadrants), where 20 of the quadrants abutted the outer walls of the field (i.e., "wall" quadrants), and 16 quadrants were displaced from the walls and comprised the interior (i.e., "open" quadrants) of the field.

Animals were placed in the center of the field. After $20 \mathrm{sec}$ had elapsed (during which the animals self-selected a "starting" location), the animals' behavior was monitored for $4 \mathrm{~min}$. Throughout this time the animal's entries into walled and open quadrants were recorded. An entry was recorded whenever both front paws crossed the border of a quadrant. Both total activity (i.e., quadrant entries regardless of category) and the percentage of entries into unwalled (open) quadrants of the field were recorded. Lastly, the fecal boli deposited by each animal was recorded as a potential measure of emotionality. It should be noted that a 4-min test was explicitly chosen because changes in exploratory behavior are negligible during this time, and thus exploratory behavior in the open field (during a 4-min test) is not likely to be appreciably modulated by learning.

\section{Balance beam}

Animals were placed on a $40 \times 0.7 \times 2 \mathrm{~cm}$ (length $\times$ width $\times$ height) beam suspended $30 \mathrm{~cm}$ above the ground. The beam was explicitly designed so that animals do not typically fall from it. Instead, movement along the beam was the variable of interest, as movement is presumed to interact with balance. In a 4-min test, mice exhibit wide variability in the amount of movement along its length.

\section{Light/dark preference}

A $10 \times 36-\mathrm{cm}$ chamber was divided across its length into two halves. One half was white and brightly lit (100 Lux), and the other half was black and dim (5 Lux). The two halves were divided by a wall, which at its base had a $4.5-\mathrm{cm}$ square opening that joined the black and white compartments. Animals were placed in the black side of the chamber and allowed to explore for $4 \mathrm{~min}$. The latency to first entry into the white chamber, percent of total time in the white chamber, and number of crossings between the black and white chambers were recorded.

\section{Rod suspension}

Animals were hung from their front paws from a 4-mm-diameter, 4-cm-long rod coated with black rubber (shrink tubing) and suspended $30 \mathrm{~cm}$ above ground. The ends of the rod terminated in vertical walls, which prohibited animals from climbing onto the top of the rod. Latency to drop from the rod (an index of front paw strength) was recorded.

\section{Pain sensitivity}

Upon being placed on a $52.6^{\circ} \mathrm{C}$ aluminum plate, animals' latency to raise a hind paw and to either lick or shake the paw served as the index of pain sensitivity.

\section{Screen hanging}

Animals were placed on the underside of a wire mesh screen (7-mm grids) tilted $40^{\circ}$ from vertical and suspended $24 \mathrm{~cm}$ from the ground. The latency to drop from the screen was recorded.

\section{Post-shock freezing}

Freezing after the offset on an unsignaled shock is often interpreted as a measure of fear. Animals were acclimated for $20 \mathrm{~min}$ to a $25-\mathrm{cm}$ square chamber (60 Lux illumination) with a stainless-steel grid floor. On the subsequent day, they were returned to the chamber, where after $10 \mathrm{~min}$ a $0.6-\mathrm{mA}, 500-\mathrm{msec}$ constantcurrent scrambled foot shock was administered through the floor. The shock was delivered upon the command of the experimenter, who initiated the shock when each animal was located near the center of the chamber with all paws on the grid floor. Using this method, the actual delivery of the shock typically occurred between 10 and $10.5 \mathrm{~min}$. During and after a brief time (500-1000 msec) following the shock, the animals exhibit a burst of activity, after which they exhibit "freezing," a presumed index of fear. The duration of freezing (the latency for each rear paw of the animal to move $2 \mathrm{~cm}$ [the span of three floor grids] after the initiation of freezing) served as the dependent variable.

\section{Exploration in a straight alley}

A straight alley was used that was $30 \mathrm{~cm}$ above ground. The alley was $260 \mathrm{~cm}$ long and $7 \mathrm{~cm}$ wide with 3-cm-high walls. The initial $29 \mathrm{~cm}$ of the alley (the "start" box) was enclosed in 12-cm-high white walls with an orange acetate ceiling. The exit from this box could be blocked with a sliding guillotine door made of clear Plexiglas. The interior of the start box was 4 Lux, and the alley beyond the start box was 40 Lux. Animals were placed in the start box with the exit blocked. After $60 \mathrm{sec}$, the door was raised and animals were allowed to explore the alley for $4 \mathrm{~min}$. The latency for each animal to cross a point in the alley $213 \mathrm{~cm}$ from the exit of the start box was recorded and served as an index of exploratory behavior.

\section{Swimming speed}

Swimming speed was estimated from the animals' performance on the first $39 \mathrm{sec}$ of Trial 1 in the water maze. (The shortest latency exhibited by any animal to reach the hidden platform on Trial 1 was 39 sec. Thus, a period of uninterrupted swimming could be obtained for $39 \mathrm{sec}$ for all animals.) The first 15 and the last $15 \mathrm{sec}$ of the $39-\mathrm{sec}$ interval were used to determine swim rates $(\mathrm{cm} / \mathrm{sec})$ for each animal. Based on a comparison of these two periods, it was possible to assess changes in swim rates as a function of time (possibly a reflection of fatigue).

\section{Body weights}

Body weights were recorded during a period of free-feeding immediately preceding the onset of behavioral testing, and during 
periods of food deprivation. As young and aged animals did not differ in their response to deprivation, only body weights during the free-feeding period were included in the statistical analysis (thus avoiding any inflation of the amount of variance accounted for by any factor on which body weights were a relevant variable).

\section{Analyses}

To compare the performance of groups, on tasks with a single test trial the two groups were compared with an independent samples $t$-test. Where multiple trials were used, a two-factor ANOVA (with trials as a repeated measure) was used to compare the groups' performance. General learning abilities were assessed with unrotated principal component analyses. For the principal component analysis, animals' performance on all tasks was assessed during acquisition. For this purpose, data for each animal on multi-trial tasks were taken from the point in the acquisition curve that was intermediate between initial performance and terminal performance, i.e., in the "middle" of the acquisition phase. As described above, the average performance of two trials served as each animal's performance on multi-trial tasks (see Matzel et al. 2003, for additional detail). We have consistently found that only performances during acquisition of the various learning tasks are positively correlated. In the tasks in which there was only one testing trial (i.e., fear conditioning and passive avoidance) we used training parameters that we have previously found to result in sub-asymptotic responding during testing.

To determine the existence of a general learning factor, first, the performance data of all (young and old) animals for the learning tasks were subjected to a principal component analysis. From this analysis, each animal was assigned a factor score (which is analogous to an average $Z$-score for each animal computed from the $\mathrm{z}$ scores obtained for that animal on each task, with the $Z$-score for each task weighted for the degree to which that task contributed to the principal factor). The factor scores of young and aged animals were compared to assess differences in general learning abilities across the two ages. (As these factor scores would later be used to assess the correlation between general learning abilities and working memory span and capacity, this initial analysis was computed only on animals' performance on the learning tasks and did not include these measures of working memory.) Subsequently, separate principal component analyses were conducted on the learning and working memory performance of young and aged animals. These analyses allowed us to assess the extent to which variable loadings changed across the life span.

Given the number of noncognitive variables examined in the present study, the sample size would generally be considered too small to permit principal component analysis of the combination of cognitive and noncognitive variables. Thus, to estimate the impact of noncognitive variables on general learning abilities, correlations were computed between factor scores indicative of general learning abilities (as described above) and each of the noncognitive performance measures. Subsequently, relevant variables (i.e., those found to correlate with general learning abilities) were further examined by comparing differences in performance on these variables in the best and worst learners in both young and old animals.

Both male and female animals contributed to these analyses. However, the number of male and female subjects was too small to assess sex $\times$ age as a separate variable in our principal component analyses. Nevertheless, it was of interest to compare the performance of male and female animals at young and old ages. To do so, the same data points that contributed to the factor analyses of age were separated by sex and subjected to a two- factor (sex $\times$ age) ANOVA to provide an estimation of the variations in cognitive performance as a function of both sex and age.

\section{Results}

\section{Task-specific learning performance}

To provide a framework with which to interpret the broader results, first we will summarize data obtained from both young and old animals (collapsing across sex) on each of six learning tasks (Lashley Maze, passive avoidance, odor discrimination, spatial water maze, fear conditioning, reinforced alternation, and the radial arm maze). These tasks are presumed to impinge on different sensory, motor, motivational, and information processing systems.

\section{Passive avoidance}

Upon stepping from a safe platform, animals were exposed to a presentation of a bright light and loud noise. The ratio of posttraining to pretraining step latencies is illustrated in the top panel of Figure 1. Both young and old animals exhibited similar increases in step latencies after a single pairing of the step with a presentation of bright light and noise, $t_{(56)}=0.11$, ns. Although young and old animals did not differ from one another, both groups exhibited a substantial increase in latency after the single training trial.

\section{Fear conditioning}

Animals received a tone (CS) paired with a mild foot shock (US). To measure the conditioned fear responses, lick rates (of waterdeprived animals) were assessed prior to and during the presentation of the tone CS. The latency to complete 25 licks prior to CS onset and the latency to complete 25 licks in the presence of the CS were recorded for each animal. The ratio of CS to pre-CS latencies is illustrated in the middle panel of Figure 1. Although both groups exhibited ratios significantly $>1.0$ (which would reflect no change in lick rates during the CS), young and old animals did not differ from one another, $t_{(56)}=0.09$, ns.

\section{Reinforced alternation}

Animals were trained to alternate between arms in a T-maze to obtain reinforcement. The percentages of correct response on Trials 6-11 were computed for each animal. This range of trials was chosen for analysis because, with our procedures, animals exhibit significant acquisition during this range, but most animals have not yet reached an asymptotic level of performance. The percentages of correct responses for Trials 6-11 are illustrated in the bottom panel of Figure 1. Young animals performed significantly better than did their aged counterparts, $t_{(56)}=2.34$, $P<0.03$.

\section{Lashley Maze}

Animals were trained to locate food in the Lashley Maze. Errors to reach the goal box across trials are depicted for young and old animals in Figure 2. Although aged animals made nominally fewer "errors" on Trial 1 (prior to any learning), they exhibited inferior performance relative to young animals across the remaining trials. This pattern of results is reflected in a significant group $\times$ trial interaction, $F_{(4,280)}=3.88, P<0.01$.

\section{Odor discrimination}

Animals were trained to locate food in one of four randomly assorted locations by using an odor cue to guide their behavior. Figure 3 illustrates the mean errors to locate the target food cup for young and old animals. Aged animals exhibited significantly more errors to locate food, $F_{(1,192)}=9.60, P<0.01$, although, by the fourth training trial, both young and old animals performed 

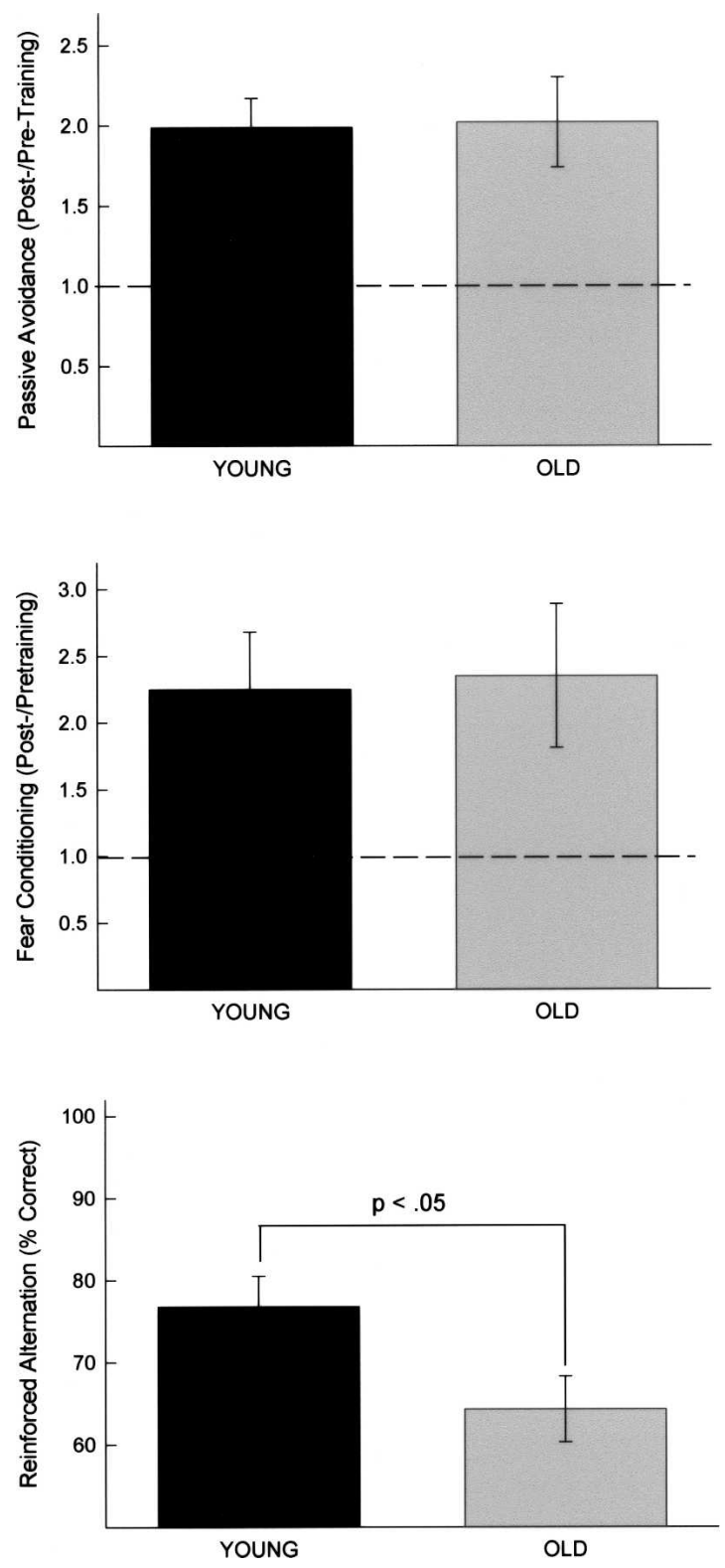

Figure 1. (Top) Upon stepping from a safe platform, animals were exposed to a presentation of a bright light and loud noise. The mean ratio of post-training to pretraining step latencies is illustrated. Brackets indicate standard errors. (Middle) Animals received a tone (CS) paired with a mild foot shock (US). To measure the conditioned fear responses, the lick rates (of water-deprived animals) were assessed prior to and during the presentation of the tone CS. The latency to complete 25 licks prior to CS onset and the latency to complete 25 licks in the presence of the CS were determined for each animal. The mean ratio of CS to pre-CS latencies is illustrated. Brackets indicate standard error. (Bottom) Animals were trained to alternate between arms in a T-maze to obtain reinforcement. The percentage of correct response on Trials 6-11 was computed for each animal. This range of trials was chosen for analysis because, with our procedures, animals exhibit significant acquisition during this range, but most animals have not yet reached an asymptotic level of performance. The mean percentages of correct responses for Trials 6-11 are illustrated. Brackets indicate standard error.

similarly, as indicated by a group $\times$ trial interaction, $F_{(1,192)}=3.14, P<0.05$. Based on this later observation, it is not likely that the learning deficit exhibited by the aged animals was a reflection of a simple olfactory impairment.

\section{Spatial water maze}

Animals were trained to locate a hidden platform submerged below the surface in a tank of opaque water. Figure 4 (top panel) illustrates the latency to locate the hidden platform recorded across each of 11 training trials. Aged animals exhibited longer latencies to locate the hidden platform across 11 training trials, $F_{(1,616)}=82.5, P<0.001$, although initial latencies did not differ significantly between young and old animals, as reflected in a group $\times$ trial interaction, $F_{(1,616)}=2.69, P<0.01$. The swimming speeds of aged animals were significantly slower than those of the young animals (see below). As this behavioral deficit would affect any measure of latency, the accuracy with which animals located the hidden platform during initial acquisition was also analyzed. Owing to a recording failure, these measures were not available for every trial and, consequently, Trials $1,4,7$, and 10 were analyzed. For those trials, a ratio of the optimal path length (the length of a straight line between the start location and the escape platform) relative to the actual path taken by each animal length was computed. (Since starting locations [and thus the length of the optimal path] differ on each trial, this measure provides a slightly more accurate index of the animals' performance than would absolute path lengths.) These measures were obtained with a video-tracking device. The results of this analysis are presented in the middle panel of Figure 4. Consistent with the latency data, aged animals exhibited deficits relative to young animals, although the aged animals ultimately obtained a level of accuracy comparable to that of the young. This effect was evident in a trial $\times$ group interaction, $F_{(3,168)}=4.79, P<0.01$.

After the 11th training trial, animals were returned to their home cages for $3 \mathrm{~h}$, and were then administered a 30-sec "probe" trial in which the escape platform was removed from the maze, and the percent of time spent searching in the target quadrant was recorded. The results of this analysis are presented in the bottom panel of Figure 4 . Although each group spent $>25 \%$ of their time searching in the target quadrant (indicative of a spatial search strategy), the percentage of time in the target quadrant was significantly different from a random search (for which it is assumed that $25 \%$ of search time will be spent in each of four quadrants) only in the young animals, $\chi_{(1)}^{2}=14.44, P<0.01$. Relatedly, young animals exhibited a tendency to spend more time in the target quadrant than did the old animals, $t_{(56)}=1.97$, $P<0.06$. In combination with the path length data (above), the results of this probe trial indicate that the young animals had implemented a spatial strategy to locate the platform. While the path length data suggest that old animals had also acquired a spatial strategy, both path length and probe trial data indicate that these animals were impaired in the acquisition and/or retention of this strategy relative to their young counterparts.

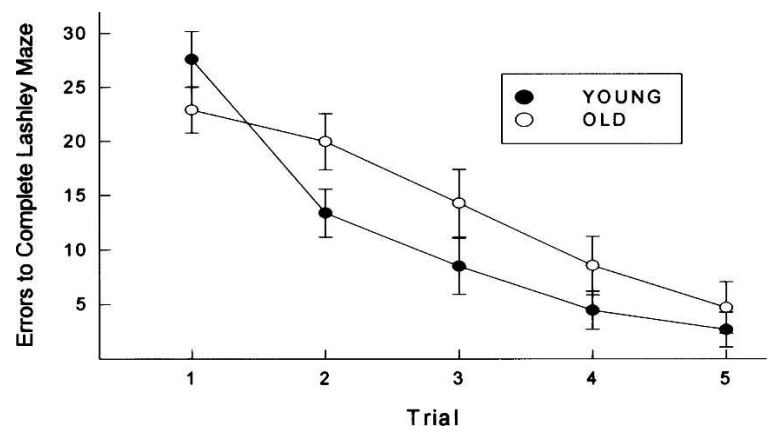

Figure 2. Animals were trained to locate food in the Lashley Maze. Mean errors to reach the goal box across trials are depicted for young and old animals. Brackets indicate standard error. 


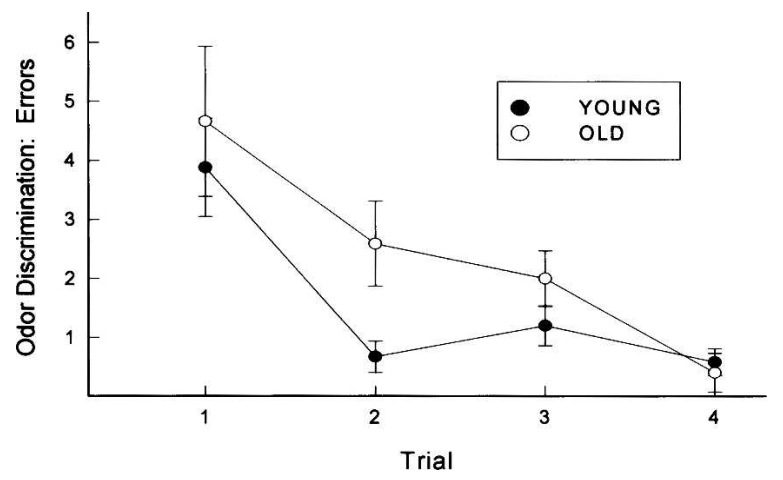

Figure 3. Animals were trained to locate food in randomly assorted locations by using an odor cue to guide their behavior. Illustrated are the mean errors to locate the target food cup for young and old animals. Brackets indicate standard error.

\section{Radial arm maze}

On each of eight initial trials (one trial per day), animals were allowed to retrieve a pellet of food at the end of each of eight arms in a radial arm maze. A reentry into an arm from which food had already been retrieved was scored as an error. Figure 5 illustrates the mean number of errors committed by young and old animals across the initial eight trials of acquisition. Across all trials, young animals committed fewer errors than old animals, $F_{(1,376)}=45.7, P<0.001$. More errors were exhibited by old animals on the first training trial (i.e., before substantial learning would have occurred), and this appeared to reflect a tendency of the old animals to perseverate in such a manner that they reentered arms from which food had just been retrieved. This pattern of perseveration was not apparent by the third training trial, although the difference in number of errors between the young and old animals persisted for the remaining five trials. Upon completion of the initial eight trials, all animals received additional training (for a total of 12 trials) in this primary maze as well as a second radial arm maze. By the 12th trial, the performance of young and aged animals did not differ in the primary maze, $t_{(56)}=1.12$, ns. This additional training insured that all animals were performing similarly prior to the initiation of tests of working memory (see below and Materials and Methods).

\section{General learning abilities}

Animals exhibited a wide range of variability in performance across tasks, although some individuals consistently performed better or worse than the median performance on all tasks, a result that is consistent with prior work (Matzel et al. 2003, 2006; Kolata et al. 2008) and indicative of a conserved influence on performance across tasks. To quantify these observations, first a principal component analysis was conducted on the acquisition performance of the entire sample of 58 animals (including 30 young and 28 old mice, collapsed across males and females) that contributed data to every learning task. The results of this analysis are presented in Table 1. (For the purpose of this analysis, reinforced alternation was excluded as the data obtained in this task are noncontinuous. Likewise [as described above], one goal of this analysis was to generate factor scores with which to describe the learning performance of individual animals and, consequently, measures of working memory span and capacity were excluded.) A principal factor was extracted that accounted for $31 \%$ of the variance across tasks. All learning tasks loaded in a consistent direction on this factor, and all variables exhibited moderate to strong loading weights. Based on this combined analysis of young and old animals, each animal was assigned a factor score. A factor score is analogous to each animal's average
$Z$-score for the six tasks, with each $Z$-score weighted by the extent to which the corresponding task contributed to the principal factor. Factor scores served to rank animals on the variable captured by the principal factor, where negative values indicated overall performance that was better than the group mean, and positive values indicated performance that was worse than the group mean. Factor scores were then separated by group (young and old), where it was determined that young animals averaged $-0.30( \pm 0.12)$ and old animals averaged $+0.29( \pm 26)$, indicative of better general learning performance in young animals, $t_{(56)}=2.11, P<0.05$ (see Fig. 6). Of note, relative to their young
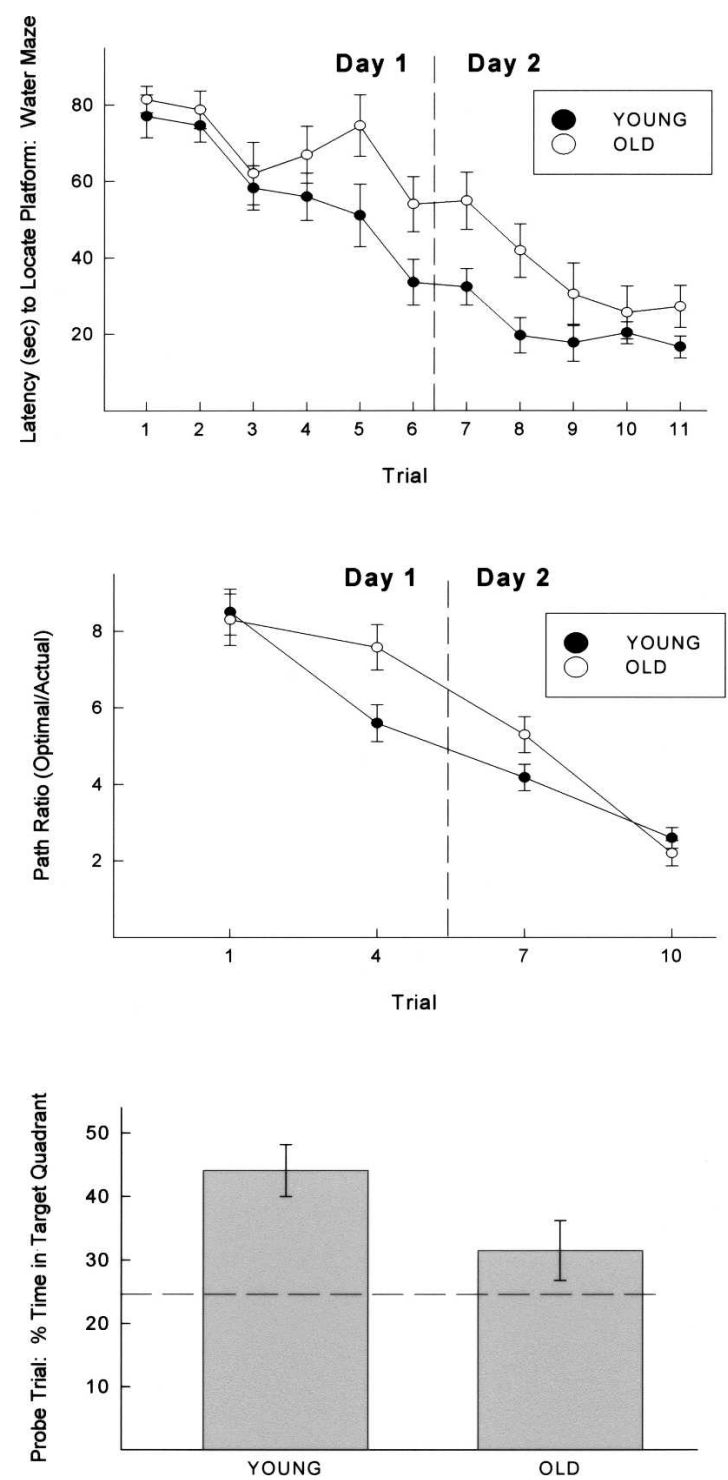

Figure 4. (Top) Animals were trained to locate a hidden platform submerged below the surface in a tank of water. Illustrated is the latency to locate the hidden platform recorded across each of 11 training trials. Brackets indicate standard error. (Middle) The accuracy (distance traveled/optimal distance) with which the animals located the hidden platform is plotted for the first, fourth, seventh, and tenth training trials. Brackets indicate standard error. (Bottom) After the 11th training trial, animals were returned to their home cages for $3 \mathrm{~h}$ and were then administered a 30-sec "probe" trial in which the escape platform was removed from the maze and the percent of time spent searching in the target quadrant was recorded. The mean time in the target quadrant is depicted. Brackets indicate standard error. 


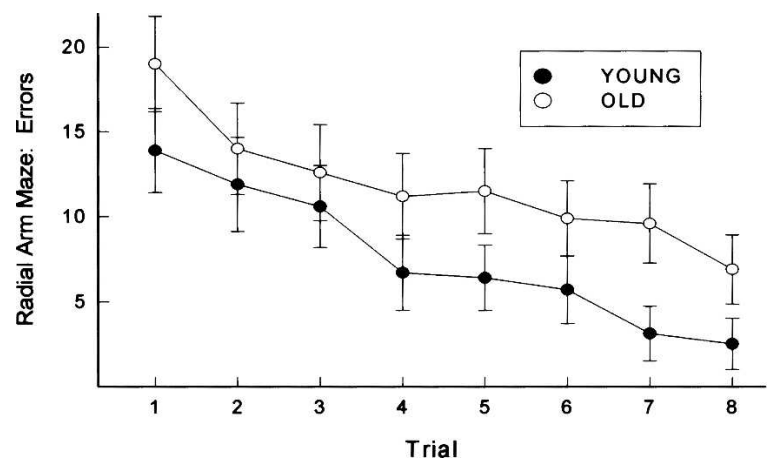

Figure 5. On each of eight initial trials (one trial per day), animals were allowed to retrieve a pellet of food at the end of each of eight arms in a radial arm maze. A reentry into an arm from which food had already been retrieved was scored as an error. Illustrated is the mean number of errors committed by young and old animals across the initial eight trials of acquisition. Brackets indicate standard error.

counterparts, old animals exhibited far more variable performance in all learning tasks, and this was reflected in the higher variability in their factor scores. This observation was confirmed by an $F$-test for heterogeneity of variance, $F_{(29,27)}=5.93, P<0.01$. To further explore this observation, the average factor scores of the top and bottom seven animals (corresponding roughly to the worst and best quartile of animals in each group) were compared, and it was determined that the best learners did not differ among the two groups, $t_{(12)}=0.97$, ns. In fact, the animal with the best aggregate performance on the learning battery was drawn from the aged sample. However, in the quartiles representing the poorest learners from each group, aged animals exhibited significantly impaired general learning abilities relative to their young counterparts, $t_{(12)}=2.55, P<0.05$. These data (illustrated in Fig. 6) suggest that, even under controlled laboratory conditions (and among genetically "homogeneous" mice), aging does not have a singular impact on general cognitive abilities. This effect is further explored below, including an analysis of the distribution of males and females in the upper and lower quartiles of learning abilities.

\section{Working memory and general learning abilities}

Upon completion of acquisition testing in the radial arm maze, all animals received additional training in the primary and secondary radial arm mazes until asymptotic performance was attained. At that point, tests of working memory span (resistance to decay) and capacity (resistance to interference) were assessed. Separate factor analyses were conducted for young and old animals, and these analyses included all of the learning variables (described above) as well as these tests of working memory. The results of these analyses are provided in Table 2 . Looking first at the performance of young animals, it was determined that both working memory span and capacity loaded on a principal factor with variables indicative of learning. Consistent with prior work (Kolata et al. 2005), working memory span loaded weakly relative to working memory capacity. In contrast, among old animals working memory span and capacity each loaded with similarly high weights. These observations were further explored by assessing the correlations between these measures of working memory and individual animals' general learning performance (i.e., factor scores obtained from the overall factor analysis described above and in Table 1). In young mice, working memory capacity and general learning abilities were significantly correlated, $r_{(28)}=0.45, P<0.01$, while working memory span and general learning abilities were not, $r_{(28)}=0.11$, ns. In contrast, in old animals working memory capacity and span were each highly correlated with general learning abilities, $r s_{(26)}=0.72$ and 0.68 (respectively), $P s<0.01$.

\section{Sex differences in task-specific performance and general learning abilities}

Contributing to the analyses above were 30 young and 28 old animals. Of the young animals, 16 were female and 14 male, while of the old animals, 16 were female and 12 were male (after the deaths of two of the aged males). The subsamples of males and females were too small to permit any useful factor analysis of those data. To assess differences in cognitive abilities across ages and sexes, the same data points that contributed to the above factor analyses were compared across these four subgroups. For each learning task, an ANOVA was computed with age and sex as factors. No differences between sexes were observed in passive avoidance, $F_{(1,54)}=0.004$, fear conditioning, $F_{(1,54)}=0.003$, the Lashley Maze, $F_{(1,54)}=0.41$, or reinforced alternation, $F_{(1,54)}=0.36$. Likewise, sex differences were not observed in tests of working memory span, $F_{(1,48)}=0.37$, or capacity, $F_{(1,48)}=0.040$. In none of these tasks was an interaction between sex and age observed, $F s_{(1,54)} \leq 0.93$.

In contrast to the absence of sex differences on the above tasks, females committed fewer errors during acquisition of the odor discrimination response, $F_{(1,54)}=5.41, P<0.03$. However, males performed better in the spatial water maze task, $F_{(1,54)}=5.42, P<0.03$, and a similar tendency for better performance by males was observed in the radial arm maze (the other explicitly spatial learning task in this battery), $F_{(1,48)}=2.63$, $P<0.11$, although this tendency did not attain statistical significance. In none of these tasks was an interaction between sex and age observed, $F s \leq 0.82$.

In summary, males and females performed similarly in most tests of learning and working memory, although females outperformed males in a task that made explicit demands on olfactory discrimination, while males appeared to outperform females in tests dependent on spatial navigation. Despite no consistent pattern of difference between males and females, it was of interest to compare their general learning abilities as a function of age. To do so, an ANOVA was computed on the aggregate performance measures (factor scores) on the six learning tasks that contributed to the factor analysis described above. No differences were detected between the sexes, $F_{(1,54)}=1.42$, and no interaction was observed between sex and age, $F_{(1,54)}=0.48$. The factor scores of aged males were nominally higher (indicative of poorer overall performance in the learning battery) than those of aged females

Table 1A. Factor loadings from a principal component analysis (unrotated) of all 58 animals ( 30 young, 28 old) of acquisition of six learning tasks

\begin{tabular}{lc}
\hline & Factor $\mathbf{1}$ \\
\hline Lashley Maze & 0.51 \\
Passive avoidance & $0.40^{\mathrm{a}}$ \\
Fear conditioning & $0.63^{\mathrm{a}}$ \\
Odor discrimination & 0.66 \\
Water maze & 0.69 \\
Radial arm maze & 0.36 \\
\hline Eigenvalue & 1.86 \\
\hline Percent variance explained & 31 \\
\hline
\end{tabular}

an the cases of passive avoidance and fear conditioning, in their raw form, higher performance scores reflect better learning, whereas for all other learning tasks, lower performance scores reflect better learning. So that all learning scores are represented with the same valance, the performance scores for passive avoidance and fear conditioning are entered into the factor analysis as inverse values. For all learning measures, lower raw scores reflect better performance. 
Table 1B. Correlations between tasks represented in Table $1 \mathrm{~A}$ (30 young and 28 old mice)

\begin{tabular}{lccccc}
\hline & $\begin{array}{c}\text { Passive } \\
\text { avoidance }\end{array}$ & $\begin{array}{c}\text { Fear } \\
\text { conditioning }\end{array}$ & $\begin{array}{c}\text { Odor } \\
\text { discrimination }\end{array}$ & $\begin{array}{c}\text { Water } \\
\text { maze }\end{array}$ & $\begin{array}{c}\text { Radial } \\
\text { arm maze }\end{array}$ \\
\hline Lashley Maze & 0.06 & $0.43^{\mathrm{a}}$ & 0.01 & 0.20 & 0.03 \\
Passive avoidance & & 0.04 & $0.32^{\mathrm{a}}$ & 0.10 & 0.04 \\
Fear conditioning & & & 0.21 & 0.23 & 0.01 \\
Odor discrimination & & & & $0.35^{\mathrm{a}}$ & 0.18 \\
Water maze & & & & 0.23 \\
\hline
\end{tabular}

${ }^{\mathrm{a}} \mathrm{P}<0.05$.

(means $=0.54 \pm 0.47$ and $0.05 \pm 0.26$, respectively). A planned comparison indicated that this difference did not reach statistical significance, $F=1.76$. This tendency is further explored below.

\section{Physical characteristics and exploratory behaviors}

In addition to learning, we assessed animals' performance on 15 tests of sensory and motor responses, emotionality, exploratory
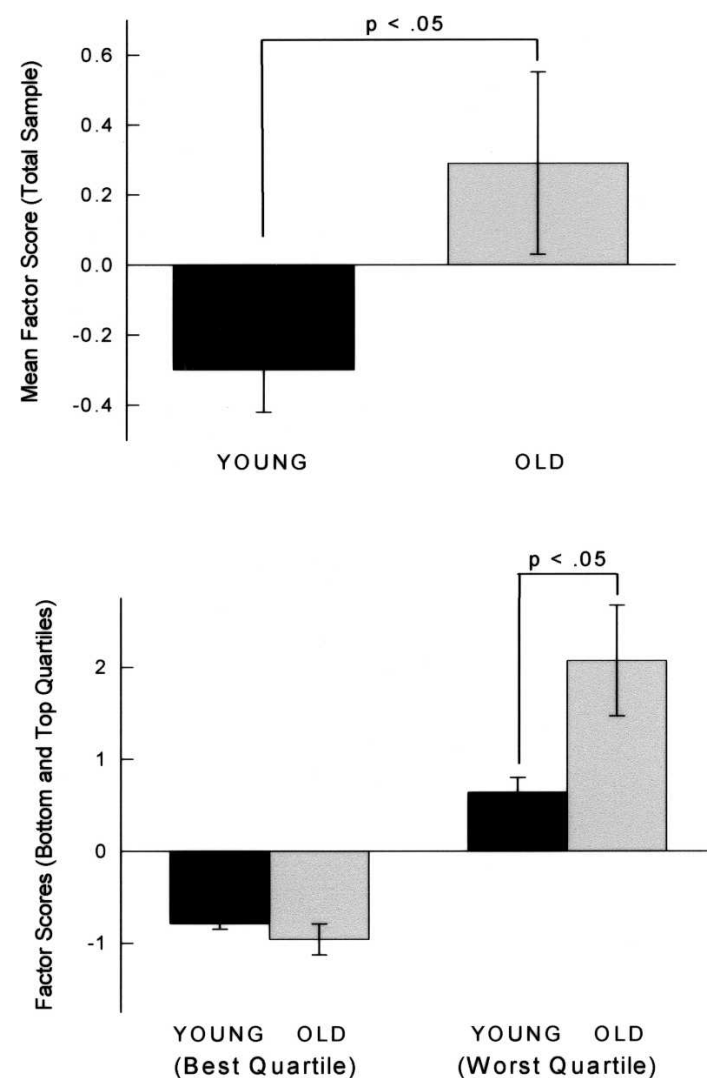

Figure 6. (Top) Based on a combined principal component analysis of young and old animals, each animal was assigned a factor score. A factor score is analogous to each animal's average Z-score for the six tasks, with each Z-score weighted by the extent to which the corresponding task contributed to the principal factor. Factor scores serve to rank animals on the variable captured by the principal factor, where negative values indicated overall performance that is better than the group mean, and positive values indicate performance that is worse than the group mean. The mean factor scores of young and old animals are illustrated. Brackets indicate standard error. (Bottom) Relative to their young counterparts, old animals exhibited far more variable performance in all learning tasks, and this was reflected in the higher variability in their factor scores. To further explore this observation, the average factor scores of the top and bottom six animals (corresponding roughly to the worst and best quartile of animals in each group) were compared. The mean performances of the best and worst learners (bottom and top quartiles) are illustrated. Brackets indicate standard error. behaviors, and fitness. Measures of exploration and emotionality included fecal boli production in a novel environment (an open field), overall activity in an open field, exploration of the unwalled areas of an open field, latency to enter the lighted compartment in a dark/light box, time spent in the lit compartment in a black/white box, and transitions between the dark and lighted compartments of a black/white box, duration of a freezing response after foot shock, and the latency to explore the length of a novel straight alley. Other measures included pain sensitivity (on a hot plate), ambulatory distance on a balance beam, the latency to fall from an elevated screen, the latency to fall from a suspended rod, swimming speeds, and body weights.

\section{Open field}

Fecal boli deposited in a 4-min test in a novel open field did not differ between groups, although aged animals exhibited a tendency to deposit more boli (means $=4.85, \pm 0.57$ and 6.17, \pm 0.65 , young and old, respectively, $t_{(56)}=1.71, P<0.10$ ). Total activity (total grid crossings) in the open field did not differ between groups (means $=72.7 \pm 9.8$ and $68.1 \pm 11.0$, young and old, respectively, $\left.t_{(56)}=0.34, \mathrm{~ns}\right)$, although old animals exhibited a tendency to make a greater percentage of grid crossings in the unwalled areas of the field (means $=9.4 \pm 2.0$ and $15.2 \pm 2.7$, young and old, respectively, $t_{(56)}=1.87, P<0.08$ ). While suggestive of the possibility that old animals were more exploratory than their young counterparts, this tendency was not observed in other measures of exploration (e.g., in the straight alley or the dark/light box). Importantly, unlike what is typically observed in young animals, in aged animals measures of exploration did not seem to be related to individual differences in general learning abilities (see below).

\section{Dark/light box}

Animals were placed in the darkened side of a novel dark/light box. The latency to first enter the lighted side of the box did not differ between groups (means $=23.8 \pm 3.66$ and $27.5 \pm 6.0$, young and old, respectively, $\left.t_{(56)}=0.54, \mathrm{~ns}\right)$. Likewise, there was no difference between groups in the number of crossings between the two compartments (means $=25.8 \pm 7.1$ and $18.2 \pm 1.2$, young and old, respectively, $t_{(56)}=1.03$, ns), or in the total time spent on the light side of the box (means $=134.8 \pm 7.1$ and $143.6 \pm 7.2$, young and old, respectively, $\left.t_{(56)}=0.73, \mathrm{~ns}\right)$.

\section{Straight alley}

Animals were placed in the start box of a straight alley and, after a 2-min period, were allowed to explore the alley. The latency to reach the end of the alley did not differ between groups (means $=152.5 \pm 18.0$ and $146.7 \pm 18.6$, young and old, respectively, $t_{(56)}=0.29$, ns).

\section{Screen hanging}

Animals were placed on the underside of a $45^{\circ}$ elevated screen, and their latency to fall from the screen was recorded. Young and old animals did not differ in their latency to fall (means $=61.3 \pm 13.9$ and $51.9 \pm 12.2$, young and old, respectively, $\left.t_{(56)}=0.44, \mathrm{~ns}\right)$.

\section{Balance beam}

Animals were placed on a narrow, elevated beam. During a 180sec test, four of 30 young animals fell from the beam, and six of 28 old animals fell. Distance traveled/min on the beam was cal- 
Table 2. Factor loadings from two independent principal component analyses (unrotated) of 30 young and 28 old animals on six learning tasks and two measures of working memory performance

\begin{tabular}{|c|c|c|}
\hline & Young & Old \\
\hline Lashley Maze & 0.66 & 0.55 \\
\hline Passive avoidance & 0.35 & 0.65 \\
\hline Fear conditioning & 0.65 & 0.72 \\
\hline Odor discrimination & 0.64 & 0.60 \\
\hline Water maze & 0.65 & 0.06 \\
\hline Radial arm maze & 0.40 & 0.58 \\
\hline Working memory span & 0.36 & 0.88 \\
\hline Working memory capacity & 0.73 & 0.81 \\
\hline Eigenvalues & 2.64 & 3.37 \\
\hline Percent variance explained & 33 & 43 \\
\hline
\end{tabular}

culated, and young animals exhibited significantly more activity, $t_{(56)}=3.5, P<0.01$.

\section{Rod hanging}

Animals were hung by their front paws from an elevated rod. Aged animals fell significantly faster than young, $t_{(56)}=2.36$, $P<0.05$.

\section{Hot plate}

Old animals exhibited significantly shorter latencies to lick a hind paw on the hot plate test for pain sensitivity, $t_{(56)}=2.09$, $P<0.05$.

\section{Shock-induced freezing}

The duration of inactivity following an unsignaled foot shock was significantly prolonged in aged animals relative to their young counterparts, $t_{(56)}=2.13, P<0.05$. This prolongation of freezing may reflect an increase in pain sensitivity, as suggested by shorter latencies to lick a hind paw when confined to the hot plate (see above).

\section{Body weights}

Aged animals were significantly heavier during periods of free feeding than were young counterparts, means $=24.0( \pm 0.87)$ and $29.6( \pm 0.65)$, young and old, respectively, $t_{(56)}=5.17$, $P<0.001$.

\section{Swimming speeds}

The swimming speeds $(\mathrm{cm} / \mathrm{sec})$ of young and old animals were compared during the first and last $15 \mathrm{sec}$ of a 38 -sec period of uninterrupted swimming. Young animals swam at a rate of 13.03 $( \pm 0.36)$ and $12.35( \pm 0.32) \mathrm{cm} / \mathrm{sec}$ during these two periods, whereas the aged animals swam at a rate of $11.36( \pm 0.27)$ and $9.67( \pm 0.24) \mathrm{cm} / \mathrm{sec}$ during the same periods. A significant difference was found between groups $F_{(1,56)}=28.54, P<0.001$, as was a significant group $\times$ time interaction, $F_{(1,56)}=15.64$, reflecting the observation that aged animals exhibited a relatively large reduction in swimming speed across the two time periods.

\section{Relationship of physical characteristics and unlearned behaviors to general learning abilities}

Of particular concern in the present work was the relationship of variations in noncognitive performance to general learning abilities. However, it is commonly asserted that a minimum of five to 10 subjects per variable is necessary to conduct a stable principal component analysis, and the number of subjects used in the present study was insufficient to conduct such an analysis. Thus, rather than employing principal component analysis, a different strategy was followed to address this issue. Table 3 presents the correlations between factor scores (indicative of general learning ability) obtained for young and old animals on the battery of learning tasks (from the analysis reported in Table 1) and all noncognitive variable described above. As is evident from Table 3 (and consistent with prior work), percent of crossings in unwalled quadrants of the open field was correlated with factor scores (indicative of general learning abilities) in the combined sample of young and old animals. However, unlike in previous work with only young animals (Matzel et al. 2006), body weights, total activity in the open field, and fecal boli deposited in the novel open field were also correlated with general learning abilities, suggesting the possibility that these noncognitive variables were differentially related to learning abilities in aged animals. Furthermore (and also in contrast to previous work), the latency to fall from an elevated screen was correlated with general learning abilities, although this relationship is quite likely a product of the relationship of body weight to cognitive performance (and we will not further attempt to interpret this effect). No other noncognitive variables were significantly correlated with factor scores, and no other consistent pattern of relationships emerged.

To better understand the relationship between those variables that were found to correlate with general learning abilities, they were further explored by comparing relevant variables (body weights, open field exploration, total activity in the open field, and fecal boli deposited in the novel open field) across the best and worst quartile of general learning among animals from the young and old samples (as previously described in Fig. 6 above). The results of this analysis are provided in Table 4 . Looking first at old animals (top panel, Table 4), it can be discerned that good and bad learners exhibited a tendency toward a difference in open field exploration (time spent in unwalled relative to walled quadrants), although good learners exhibited significantly more general activity in the open field. Notably, body weights were significantly higher among those old animals that exhibited the worst general learning abilities. Whether or not a causal relationship exists between body weight and activity cannot be ascertained from the present data, although such a relationship is certainly plausible. Importantly, the same pattern of differences was not observed in young animals (here or in previous work; Matzel et al. 2006). As would be anticipated from prior work, significantly more exploration in the open field (percent

Table 3. Correlations between factor scores of young and old animals (indicative of general cognitive ability) obtained from the principal component analysis of learning tasks summarized in Table $1 A$ and performance on measures of noncognitive tests of sensory/motor function, exploration, and emotionality

\begin{tabular}{lc} 
& Correlation with factor scores \\
\hline Open field total activity & $0.85^{\mathrm{b}}$ \\
Body weight & $0.53^{\mathrm{b}}$ \\
Open field fecal boli & $0.43^{\mathrm{b}}$ \\
Open field, \% unwalled activity & $0.34^{\mathrm{c}}$ \\
Light/dark, latency to light & 0.22 \\
Swim speed (time 2) & 0.21 \\
Rod hanging & 0.20 \\
Balance beam, distance & 0.19 \\
Swim speed (time 1) & 0.15 \\
Shock-induced freezing & 0.00 \\
Pain sensitivity & -0.05 \\
Straight alley, latency to end & -0.11 \\
Light/dark, crossings & -0.12 \\
Light/dark, time in light & -0.25 \\
Screen hanging, latency to fall & $-0.42^{\mathrm{b}}$ \\
\hline
\end{tabular}

a Factor scores were derived from the principal component analysis of learning tasks summarized in Table 1.

b $p<0.001$.

${ }^{c} P<0.05$. 
Table 4. Comparisons of variables of interest (based on principal component analysis) across the best $(n=7)$ and worst $(n=7)$ learners among aged animals

\begin{tabular}{|c|c|c|c|}
\hline Old Animals & Best learners & Worst learners & $t$-values \\
\hline $\begin{array}{l}\text { Factor scores }{ }^{\mathrm{a}} \\
\text { Body weight }(\mathrm{g}) \\
\text { Open field ( } \% \text { unwalled) } \\
\text { Open field (total activity) } \\
\text { Open field boli }\end{array}$ & $\begin{array}{r}-1.13 \pm 0.44 \\
24.11 \pm 1.74 \\
24.60 \pm 8.13 \\
78.86 \pm 2.44 \\
5.83 \pm 1.03\end{array}$ & $\begin{array}{r}2.03 \pm 0.60 \\
30.24 \pm 1.81 \\
8.30 \pm 3.46 \\
36.50 \pm 12.0 \\
5.16 \pm 2.17\end{array}$ & $\begin{array}{l}t_{(12)}=2.43, P<0.05 \\
t_{(12)}=1.84, P<0.10 \\
t_{(12)}=2.28, P<0.05 \\
t_{(12)}=0.30, \mathrm{~ns}\end{array}$ \\
\hline Young Animals & Best learners & Worst learners & $t$-values \\
\hline $\begin{array}{l}\text { Factor scores }^{\mathrm{a}} \\
\text { Body weight ( } \mathrm{g} \text { ) } \\
\text { Open field ( } \% \text { unwalled) } \\
\text { Open field (total activity) } \\
\text { Open field boli }\end{array}$ & $\begin{aligned}-0.93 & \pm 0.09 \\
25.30 & \pm 2.76 \\
22.78 & \pm 6.20 \\
78.86 & \pm 2.44 \\
6.0 & \pm 1.49\end{aligned}$ & $\begin{aligned} 0.74 & \pm 0.19 \\
24.26 & \pm 2.01 \\
1.25 & \pm 0.75 \\
67.66 & \pm 10.28 \\
7.16 & \pm 1.19\end{aligned}$ & $\begin{array}{l}t_{(12)}=0.33, \mathrm{~ns} \\
t_{(12)}=3.77, P<0.01 \\
t_{(12)}=0.09, \mathrm{~ns} \\
t_{(12)}=0.67, \mathrm{~ns}\end{array}$ \\
\hline
\end{tabular}

${ }^{a}$ Factor scores (derived from the principal component analysis summarized in Table 1) provide an estimation of animals' general learning ability, where values below zero reflect overall performance better than the group median, and values above zero reflect overall performance that is worse than the group median. On all variables, animals were divided into the best and worst general learning abilities (the top and bottom $\sim 25 \%$ of factor scores). Presented values are means \pm standard errors. Since factor scores were obtained from the top and bottom of the range of scores, a statistical comparison of factor scores was not justified; however, inspection of standard errors indicates a good separation of the best and worst learners.

activity in the unwalled quadrants) was observed in the good learners. However, no differences were detected between the best and worst learners among young animals in either total activity or body weights. Thus, an increase in body weight and a decline in general activity appear to be specifically related to general cognitive declines associated with aging.

Since male mice are typically heavier than females, and body weight was here found to be inversely related to general learning abilities in aged animals, it was of interest to explore the distribution of sexes in the subgroups of best and worst learners. Of the seven animals which comprised the best quartile of learning abilities, four were females and three were males. Of the seven animals comprising the worst quartile of learning abilities, three were females and four were males. Thus, although body weight appears to be inversely related to general cognitive abilities in aged animals, this effect does not appear to be attributable to weight as a function of sex. This result is consistent with the comparison above of the factor scores of male and female animals, which indicated no difference in the general cognitive abilities of the two sexes of aged animals. It should be noted that, since not all animals in the aged sample developed general cognitive deficits, we cannot conclusively state that sex differences in cognitive abilities will not develop with age, only that no such difference emerged at the age tested here.

\section{Discussion}

Aged animals exhibited marked deficits in the acquisition of a wide variety of learned responses representing different learning processes (i.e., "domains"), sensory, motor, and motivational requirements, and, presumably, neuroanatomical dependence. Deficits were observed in reinforced alternation, spatial water maze, Lashley Maze, radial arm maze, odor discrimination, and tests of working memory span and capacity. Given that at least two of the tasks (the water maze and radial arm maze) in this cognitive test battery were specifically designed to impinge on spatial abilities, the deficits among aged animals in this "spatial domain" are consistent with previous observations in both aging rodents (e.g., Barnes 1979; Bach et al. 1999) and humans (e.g., Newman and Kaszniak 2000). In contrast, aged animals did not differ from their young counterparts in the acquisition of a fear conditioned response or passive avoidance. Based on further analyses, the normal "learning" exhibited in these later two tasks may have arisen as a consequence of heightened fear or pain sensitivity in the old animals. Alternatively, the "normal" learning on these two tasks may have reflected the tendency for passivity in old animals (which in turn may have promoted a response pattern that was compatible with the responses indicative of learning). Also consistent with this data is the possibility that the learning processes that underlie passive avoidance and fear conditioning are themselves unaffected by age. While it is not possible to make this determination from the present data, it is notable that deficits in fear conditioning among old mice have been observed elsewhere (Feiro and Gould 2005; Gould and Feiro 2005), suggesting that the absence of a deficit here may reflect procedural variations rather than an insensitivity of this domain to the detrimental impacts of aging. This later point is of some interest, as it suggests the possibility that minor procedural variations may be sufficient to overcome some of the detrimental effects of aging on at least some cognitive tasks.

Based on a combined principal component analysis of the learning performance of young and old animals, each animal in this study was assigned a factor score (analogous to an average $Z$-score of an animal's performance across all tasks), and this score served to characterize the general learning abilities of each animal relative to the others in this sample. A comparison of the factor scores of young and old animals indicated significantly impaired general learning abilities in aged animals. However, the factor scores of the old animals were more variable than those of the young, and this observation was consistent with the higher variability of the old animals on individual learning tasks. Likewise, the primary factor in our principal component analysis of learning performance (Table 2) accounted for more of the variability in old relative to young animals. This later observation suggests that aging adds variability to common core performance (presumably general learning ability) and that the increase in variability of individual tasks is attributable to increasing variability in general learning abilities. It is notable that this increased variability in the performance of aged animals was not attributable to the emergence of a difference between sexes as animals aged. This conclusion is based on the observation that no significant difference in factor scores existed between male and female animals in either the young or old samples.

To further assess the implications of increased variability in general cognitive ability in the aged sample, a comparison was made of the upper and lower quartile (corresponding to the worst and best overall performers) of animals from the young and old samples. Among the best quartile of learners, young and old animals did not differ and, in fact, the single best general learning ability across groups was expressed by an animal from the aged sample. However, in the quartile of worst learners, young and old animals differed significantly, i.e., more old animals were represented in the end of the distribution indicative of poor general learning abilities. This observation suggests one of two possibilities. First, the general cognitive performance of some percentage of old animals may be insensitive to the impact of aging. Alternatively (but relatedly), aging may impact cognition at different rates across animals, such that the impact of aging on general 
cognitive abilities may be slow to emerge in some subsample of aging animals. Were the latter true, one could expect that all animals would at some age exhibit a decline in general cognitive performance. The animals in the present study were $\sim 20-22 \mathrm{mo}$ of age at the time of testing, an age regarded as analogous to an "elderly" human (Crawley 2000). In fact, these animals exhibit $25 \%$ mortality by 18.8 mo of age, and $50 \%$ mortality by $25.5 \mathrm{mo}$ of age (Jackson Laboratories, Bar Harbor, ME, technical report). Although it is not possible from the present data to determine if all animals will at some age express age-related cognitive decline, it is clear from the present study that, even at this late age (at a time when the expected mortality rate is between $25 \%$ and $50 \%$ ), some percentages of animals are spared the impact of aging on cognition. However, it is notable that, at the extreme ends of human lifespan (e.g., $>86 \mathrm{yr}$ of age), cognitive decline accumulates rapidly (e.g., Kuningas et al. 2007). This observation increases the likelihood that impaired general learning abilities would be observed in increasingly greater proportion of mice were the age of our sample extended beyond the 22 mo used in the present study.

Tests of sensory/motor function, emotionality, exploration, and fitness suggested some differences between young and old animals. Old animals tended to be less exploratory than their young counterparts, and exploration was less systematically related to learning (and general cognitive abilities) in the aged subjects. Likewise, the aged animals exhibited impairments in balance and strength, and appeared more reactive to painful stimuli, but none of these variables could account for the impairment of general cognitive abilities in aged animals. In fact, an increase in pain sensitivity and/or fear may promote learning in certain tasks and, as such, may account for the lack of learning deficits observed here in the fear conditioning and passive avoidance tasks. Notably, body weight and general activity were related to general learning abilities in aged animals, i.e., the aged animals in the lower quartile of general learning abilities were heavier and less active than aged animals in the upper quartile of general learning abilities, a pattern of relationships that was not observed in young animals. This raises the possibility that body weight and/or activity may contribute to the variations in cognitive decline seen in aging populations, a possibility that has been suggested previously (Markowska and Savonenko 2002b). This speculation is consistent with suggestions that weight gain is often associated with cognitive decline (Hendrickx et al. 2005). However, the causal relationship between these variables has been complicated by the observation that caloric restriction can have a negative impact on cognitive processes (Bagger et al. 2004; Yanai et al. 2004). Given the nature of the present study, it is not possible to specify the underlying basis of the relationship between these variables, although the methods described here will provide a useful framework for future efforts in this regard.

In previous work with young animals, it has been determined that processing components of working memory (i.e., working memory capacity and selective attention) are critically related to animals' general cognitive abilities, whereas the storage components of working memory (i.e., working memory span and short-term memory) are not (Kolata et al. 2005, 2007). This observation is consistent with the suggestion from studies of humans that an individual's capacity for processing information in working memory is a potential source of variance in "intelligence" (Conway and Engle 1996; Engle et al. 1999). Consistent with these prior observations, here it was determined that, in young animals, working memory capacity (i.e., resistance to interference) loaded consistently with learning variables on a primary factor and was significantly correlated with general learning abilities, while working memory span did not. A very different pattern was observed in aged animals, where both working memory capacity and span were highly correlated with general learning abilities, and both aspects of working memory loaded more heavily with learning performance on a primary factor. The most parsimonious explanation for this increasing covariation of general cognitive abilities and working memory span is that our measure of working memory capacity (simultaneous performance in two tasks that require working memory) makes some demands on working memory span (i.e., the delay between performance on the two working memory tasks). In young animals, this reliance on working memory span is likely insufficient to tax that resource (Kolata et al. 2005). However, perturbations of working memory span in aged animals result in this resource impacting working memory capacity to an extent not observed in young subjects. This scenario assumes that the increasing reliance of general learning abilities on working memory span in aging animals is a reflection of the impact of working memory span on working memory capacity. This interpretation is consistent with previous observations by Bimonte et al. (2003), who reported that aged rats were impaired in a task that impinged on multiple components of working memory. However, these deficits increased across training trials, suggesting that they were impacted by proactive interference, an effect that is heavily dependent on working memory capacity and/or selective attention (for related results, see Markowska and Savonenko 2002a; Winocur and Hasher 2004). Alternatively, it is possible that, among aged animals, general cognitive abilities become specifically dependent on working memory span. However, such a conclusion would require a fundamental change in the nature of general cognitive processes relative to young animals and is not easily reconciled with available data or theory.

In summary, in the present work we have characterized the general cognitive declines of laboratory mice. These cognitive declines were associated with perturbations of both processing and storage aspects of working memory and, furthermore, were associated with increases in body weight and decreases in general levels of activity. Despite the homogeneous stock from which these animals were selected, aged animals exhibited marked variations in the development of cognitive impairments, such that a subsample of the aged animals were spared the impact of aging on general cognitive processes. These observations should provide a basis with which to begin to elucidate the underlying determinants of these variations in the expression of age-related cognitive declines.

\section{Acknowledgments}

This work was supported by grants from the National Institute of Aging (PHS AG022698 and AG029289) and the Busch Foundation to L.D.M.

\section{References}

Bach, M.E., Barad, M., Son, H., Zhuo, M., Lu, Y.F., Shih, R., Mansuy, I., Hawkins, R.D., and Kandel, E.R. 1999. Age-related defects in spatial memory are correlated with defects in the late phase of hippocampal long-term potentiation in vitro and are attenuated by drugs that enhance the cAMP signaling pathway. Proc. Natl. Acad. Sci. 96: $5280-5285$.

Bagger, Y.Z., Tanko, L.B., Alexandersen, P., Qin, G., and Christiansen, C. 2004. The implications of body fat mass and fat distribution for cognitive function in elderly women. Obes. Res. 9: 1519-1526.

Barnes, C.A. 1979. Memory deficits associated with senescence: A neurophysiological and behavioral study in the rat. J. Comp. Physiol. Psychol. 93: 74-104.

Barnes, C.A. and McNaughton, B.L. 1985. An age comparison of the rates of acquisition and forgetting of spatial information in relation to long-term enhancement of hippocampal synapses. Behav. Neurosci. 99: 1040-1048.

Bimonte, H.A., Nelson, M.E., and Granholm, A.E. 2003. Age-related deficits as working memory load increases: Relationships with growth factors. Neurobiol. Aging 24: 37-48. 
Conway, A.R. and Engle, R.W. 1996. Individual differences in working memory capacity: More evidence for a general capacity theory. Memory 4: 577-590.

Crawley, N.J. 2000. The laboratory mouse. Academic Press, New York.

Engle, R.W., Tuholski, S.W., Laughlin, J.E., and Conway, A.R. 1999. Working memory, short-term memory, and general fluid intelligence: A latent-variable approach. J. Exp. Psychol. Gen. 128: 309-331.

Feiro, O. and Gould, T.H. 2005. The interactive effects of nicotinic and muscarinic cholinergic receptor inhibition on fear conditioning in young and aged C57BL/6 mice. Pharmacol. Biochem. Behav. 80: $251-262$.

Gage, F.H., Dunnett, S.B., and Björklund, A. 1984. Spatial learning and motor deficits in aged rats. Neurobiol. Aging 5: 43-48.

Gallagher, M. and Rapp, P.R. 1997. The use of animal models to study the effects of aging on cognition. Annu. Rev. Psychol. 48: 339-370.

Gould, T.H. and Feiro, O. 2005. Age-related deficits in the retention of memories for cued fear conditioning are reversed by galantamine treatment. Behav. Brain Res. 165: 160-171.

Grossman, H.C., Hale, G., Light, K., Kolata, S., Townsend, D.A., Goldfarb, Y., Kusnecov, A., and Matzel, L.D. 2007. Pharmacological modulation of stress reactivity dissociates general learning ability from the propensity for exploration. Behav. Neurosci. 121: $949-964$

Hendrickx, H., McEwen, B.S., and Ouderaa, F. 2005. Metabolism, mood and cognition in aging: The importance of life style and dietary intervention. Neurobiol. Aging 26: 1-5.

Kolata, S., Light, K., Townsend, D.A., Hale, G., Grossman, H., and Matzel, L.D. 2005. Variations in working memory capacity predict individual differences in general learning abilities among genetically diverse mice. Neurobiol. Learn. Mem. 84: 242-246.

Kolata, S., Light, K., and Matzel, L.D. 2007. Selective attention is the primary determinant of the relationship between working memory and general learning abilities. Learn. Mem. 14: 22-28

Kolata, S., Light, K., and Matzel, L.D. 2008. Domain-specific and domain-general learning factors are expressed in genetically heterogeneous CD-1 mice. Intelligence (in press). doi: 10.1016/j.intell.2007.12.001.

Kuningas, M., de Rijk, R.H., Westendorp, R.G.J., Jolles, J., Slagboom, P.E., and van Heemst, D. 2007. Mental performance in old age dependent on cortisol and genetic variance in the mineralocorticoid and glucocorticoid receptors. Neuropsychopharmacology 32: 1295-1301.

Markowska, A.L. and Breckler, S.J. 1999. Behavioral biomarkers of aging: Illustration of a multi-variate approach for detecting age-related cognitive changes. J. Gerontol. 54: B549-B566.

Markowska, A.L. and Savonenko, A.V. 2002a. Protective effect of practice on cognition during aging: Implications for predictive characteristics of performance and efficacy of practice. Neurobiol. Learn. Mem. 78: 294-320.

Markowska, A.L. and Savonenko, A. 2002b. Retardation of cognitive aging by life-long diet restriction: Implications for genetic variance. Neurobiol. Aging 23: 75-86.

Markowska, A.L., Koliatsos, V.E., Breckler, S.J., Price, D.L., and Olton, D.S. 1994. Human nerve growth factor improves spatial memory in aged but not in young rats. J. Neurosci. 14: 4815-4824.

Matzel, L.D., Han, Y.R., Grossman, H., Karnik, M.S., Patel, D., Scott, N., Specht, S.M., and Gandhi, C.C. 2003. Individual differences in the expression of a "general" learning ability in mice. J. Neurosci. 23: 6423-6433.

Matzel, L.D., Townsend, D.A., Grossman, H., Han, Y.R., Hale, G., Zappulla, M., Light, K., and Kolata, S. 2006. Exploration in outbred mice covaries with general learning abilities irrespective of stress reactivity, emotionality, and physical attributes. Neurobiol. Learn. Mem. 86: 228-240.

Meliska, C.J., Burke, P.A., Bartke, A., and Jensen, R.A. 1997. Inhibitory avoidance and appetitive learning in aged normal mice: Comparison with transgenic mice having elevated plasma growth hormone levels. Neurobiol. Learn. Mem. 68: 1-12.

Morris, R.G.M. 1981. Spatial localization does not require the presence of local cues. Learn. Motiv. 12: 239-261.

Nalbantoglu, J., Tirado-Santiago, G., Lahsaini, A., Poirier, J., Goncalves, O., Verge, G., Momoli, F., Welner, S.A., Massicotte, G., Julien, J.P., et al. 1997. Impaired learning and LTP in mice expressing the carboxy terminus of the Alzheimer amyloid precursor protein. Nature 387: 500-505.

Newman, M.C. and Kaszniak, A.W. 2000. Spatial memory and aging: Performance on a human analog of the Morris Water Maze. Aging Neuropsychol. Cogn. 7: 86-93.

Plomin, R. 1999. Genetics and general cognitive ability. Nature (Suppl.) 402: C25-C29.

Plomin, R. and Spinath, F.M. 2002. Genetics and general cognitive ability $(g)$. Trends Cogn. Sci. 6: 169-176.

Rosenzweig, E.S. and Barnes, C.A. 2003. Impact of aging on hippocampal function: Plasticity, network dynamics, and cognition. Prog. Neurobiol. 69: 143-179.

Salthouse, T.A., Atkinson, T.M., and Berish, D.E. 2003. Executive function as a potential mediator of age-related cognitive decline in normal adults. J. Exp. Psychol. Gen. 132: 566-594.

Sara, S.J., Roullet, P., and Przybyslawski, J. 1999. Consolidation of memory for odor-reward association: $\beta$-Adrenergic receptor involvement in the late phase. Learn. Mem. 6: 88-96.

Sternberg, R.J. 1997. Intelligence and life-long learning. Am. Psychol. 52: 1134-1139.

Sternberg, R.J. and Kaufman, J.C. 1998. Human abilities. Annu. Rev. Psychol. 49: 479-502.

Vogel, R.W., Ewers, M., Ross, C., Gould, T.J., and Woodruf-Pak, D.S. 2002. Age-related impairment in the 250-millisecond delay eyeblink classical conditioning procedure in C57BL/6 mice. Learn. Mem. 9: $321-336$.

Winocur, G. and Hasher, L. 2004. Age and time of day effects on learning and memory in a non-matching-to-sample test. Neurobiol. Aging 25: 1107-1115.

Yanai, S., Okaichi, Y., and Okaichi, H. 2004. Long-term dietary restriction causes negative effects on cognitive functions in rats. Neurobiol. Aging 25: 325-332.

Received February 12, 2008; accepted in revised form July 1, 2008. 


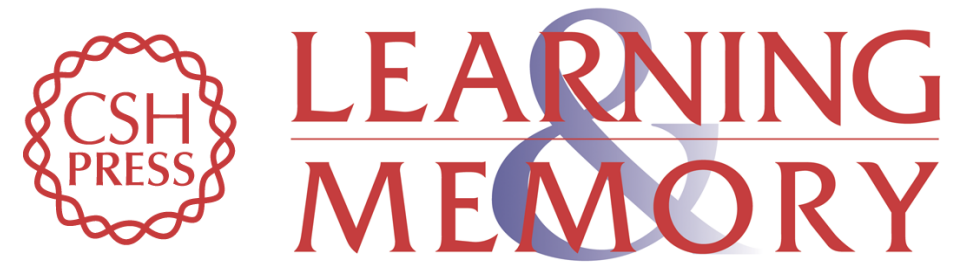

\section{Age-related declines in general cognitive abilities of Balb/C mice are associated with disparities in working memory, body weight, and general activity}

Louis D. Matzel, Henya Grossman, Kenneth Light, et al.

Learn. Mem. 2008, 15:

Access the most recent version at doi:10.1101//m.954808

References This article cites 36 articles, 6 of which can be accessed free at:

http://learnmem.cshlp.org/content/15/10/733.full.html\#ref-list-1

License

Email Alerting Receive free email alerts when new articles cite this article - sign up in the box at the Service top right corner of the article or click here. 